\title{
Processo germinativo de sementes de paineira sob estresses hídrico e salino
}

\author{
Silmara Cristina Fanti ${ }^{(1)}$ e Sonia Cristina Juliano Gualtieri de Andrade Perez ${ }^{(1)}$
}

(1)Universidade Federal de São Carlos, Dep. de Botânica, Caixa Postal 676, CEP 13565-905 São Carlos, SP. E-mail: pscf@iris.ufscar.br, dscp@power.ufscar.br

Resumo - O ambiente mutável provoca múltiplos estresses, que podem limitar a germinação da semente e a emergência e sobrevivência da plântula. Os objetivos deste trabalho foram determinar o efeito dos estresses hídrico e salino na viabilidade e vigor de sementes de paineira (Chorisia speciosa St. Hil.) e o limite máximo de tolerância a esses estresses. Para avaliação do efeito do estresse hídrico foram utilizadas soluções de manitol e PEG 6000; para o estresse salino, soluções de $\mathrm{NaCl}, \mathrm{KCl}$ e $\mathrm{CaCl}_{2}$. Após a punção do tegumento, quatro repetições de 50 sementes por tratamento foram submetidas à germinação em substrato de papel-filtro umedecido com as soluções de diferentes potenciais osmóticos e incubadas a $27^{\circ} \mathrm{C}$. As sementes apresentaram menor tolerância ao estresse hídrico simulado com PEG 6000 em relação ao simulado com manitol. O limite máximo de tolerância à seca está situado entre -0,6 e -0,7 MPa de PEG 6000 e entre -1,4 e -1,6 MPa de manitol. Independentemente dos sais utilizados, a porcentagem de germinação decresceu com a diminuição do potencial osmótico do meio. O limite máximo de tolerância ao estresse salino foi o mesmo para todos os sais avaliados e está situado entre $-1,0$ e -1,2 MPa. A paineira pode ser classificada como glicófita, com moderada tolerância aos sais $\mathrm{NaCl}, \mathrm{KCl}$ e $\mathrm{CaCl}_{2}$.

Termos para indexação: Chorisia speciosa, vigor, tolerância salina, pressão osmótica.

\section{Seed germination of paineira under water and salt stress}

\begin{abstract}
Multiple stresses promoted by a changeable environment can limit germination process, seedling emergence and survival. The objective of this work was to determine the effect of water and salt stresses on the viability and vigour of "paineira" seeds (Chorisia speciosa St. Hil.) and the maximal tolerance limits. Water stress was evaluated using mannitol and PEG 6000 solutions. Salt stress was evaluated using $\mathrm{NaCl}, \mathrm{KCl}$ and $\mathrm{CaCl}_{2}$ solutions. After coat punction, four replicates of 50 seeds for each treatment were submitted to germination on filter paper moistened with test solutions at different osmotic potential and kept at $27^{\circ} \mathrm{C}$. The lowest tolerance limit to water stress was observed when PEG solutions were used. The maximal tolerance limit was between - 0,6 and -0.7 MPa for PEG 6000 and between -1.4 and -1.6 MPa for mannitol solutions. The germination percentage decreased with the reduction of the osmotic potential of the environment, independently of the used salts. The same tolerance limits to different salts were observed between -1.0 and -1.2 MPa. Paineira may be considered a glycophyte with moderate tolerance to the salts $\mathrm{NaCl}, \mathrm{KCl}$ and $\mathrm{CaCl}_{2}$.
\end{abstract}

Index terms: Chorisia speciosa, vigour, salt tolerance, osmotic pressure.

\section{Introdução}

As plantas estão sujeitas a condições de múltiplos estresses que limitam o seu desenvolvimento e suas chances de sobrevivência, onde quer que elas cresçam. Um dos métodos mais difundidos para a determinação da tolerância das plantas aos estresses hídrico e salino é a observação da capacidade germinativa das sementes nessas condições (Larcher, 2000).
De acordo com Bewley \& Black (1994), a primeira etapa da germinação se processa com a absorção de água pela semente, mediante embebição. A velocidade de absorção da água é determinada pela sua disponibilidade, pela composição química da semente, permeabilidade do tegumento, temperatura e qualidade fisiológica da semente. A absorção de água promove a reidratação dos tecidos e, conseqüentemente, a intensificação da respiração e de todas as outras atividades 
metabólicas, que culminam com o fornecimento de energia e nutrientes necessários para a retomada de crescimento por parte do eixo embrionário (Carvalho \& Nakagawa, 2000).

O período inicial de embebição é crítico para a germinação. Assim, potenciais hídricos bastante negativos impedem a absorção de água, inviabilizando a sequência de eventos do processo germinativo (Torres et al., 1999). Por sua vez, a absorção de água pela semente é muito rápida em condição de excesso de umidade no solo, ocorrendo desorganização das membranas celulares, rupturas nos tecidos, perda de solutos para o meio e surgimento de plântulas anormais e pouco vigorosas (Lucca \& Reis, 1995)

A fim de serem simuladas condições padronizadas de estresse hídrico em condições de laboratório, estudos de germinação têm sido realizados com a utilização de soluções aquosas de manitol e polietilenoglicol, por serem compostos químicos inertes e não tóxicos (Hardegree \& Emmerich, 1994).

A inibição do crescimento ocasionada pela salinidade segundo Tobe et al. (2000), se deve tanto ao efeito osmótico, ou seja, à seca fisiológica produzida, como ao efeito tóxico, resultante da concentração de íons no protoplasma. As sementes são sensíveis aos efeitos da salinidade e, quando semeadas em soluções salinas, observa-se inicialmente uma diminuição na absorção de água (Ferreira \& Rebouças, 1992).

A resistência à salinidade é descrita como a habilidade de evitar, por meio de uma regulação salina, que excessivas quantidades de sal provenientes do substrato alcancem o protoplasma e também, de tolerar os efeitos tóxicos e osmóticos associados ao aumento da concentração de sais (Larcher, 2000). O aumento da concentração de compostos como prolina, polióis e açúcares, serve para manter o potencial osmótico da célula compatível com a manutenção da estabilidade de algumas macromoléculas, proporcionando redução na perda de atividade enzimática ou da integridade da membrana, que ocorrem quando existe estresse hídrico ou salino (Freire, 2000).

Segundo Mayer \& Poljakoff-Mayber (1989), plantas com baixa tolerância à salinidade nos vários estádios de desenvolvimento, incluindo a germinação, são denominadas glicófitas e as mais tolerantes, halófitas. Uma característica importante das halófitas é que suas sementes permanecem dormentes, sem perda de viabilidade, em altas concentrações salinas, e depois, germinam prontamente quando a concentração de sal é reduzida.
Estima-se que aproximadamente um terço das terras do planeta seja árida ou semi-árida, sendo que cerca da metade dessa área é afetada pela salinização (Serrato Valenti et al., 1991; Cavalcante \& Perez, 1995). No Brasil, essas áreas estão situadas principalmente no semi-árido da região Nordeste (Freire, 2000). A proporção de solos salinizados está aumentando em virtude do emprego incorreto de técnicas agrícolas, como adubação excessiva e irrigação com água imprópria para tal finalidade, transformando terras férteis e produtivas em terras impróprias para a agricultura. Entre os elementos que contribuem para a salinização dos solos, estão o $\mathrm{Ca}, \mathrm{Mg}, \mathrm{Na}, \mathrm{K}, \mathrm{Cl}, \mathrm{S}$ e o íon carbonato (Agboola, 1998; Freire, 2000)

Chorisia speciosa St. Hil. pertence à família Bombacaceae e ocorre em uma ampla área, que abrange, principalmente, as florestas mesófilas semidecíduas nos estados brasileiros de São Paulo, Minas Gerais, Goiás, Mato Grosso do Sul, Paraná, Santa Catarina e Rio Grande do Sul. Além da importância ecológica e ornamental, a madeira da paineira pode ser empregada na confecção de canoas, cochos, forros de móveis, na caixotaria e no fabrico de pasta celulósica (Lorenzi, 1992; Carvalho, 1994).

Este trabalho teve como objetivo verificar os efeitos dos estresses hídrico e salino na viabilidade e vigor de sementes de Chorisia speciosa St. Hil., bem como definir os limites máximos de tolerância a esses estresses.

\section{Material e Métodos}

Este trabalho foi realizado no laboratório de Ecofisiologia da Germinação de Sementes do Departamento de Botânica da Universidade Federal de São Carlos (UFSCar), SP, em março de 2001. Foram utilizadas sementes de paineira Chorisia speciosa St. Hil., provenientes de Maristela, SP, fornecidas pelo Instituto de Pesquisa e Estudos Florestais de Piracicaba (IPEF/ Esalq/USP). As sementes com teor de umidade de $11 \%$ foram mantidas em embalagens de polietileno transparente, folha dupla, à temperatura de $5^{\circ} \mathrm{C}$.

No preparo das soluções, utilizou-se água destilada acrescida de 2 ppm de Captan (Clarck \& Scott, 1982). As sementes foram selecionadas por tamanho, coloração e estado de conservação e submetidas à punção do tegumento (Fanti, 2001).

Quatro repetições de 50 sementes, usadas em cada potencial osmótico avaliado, foram dispostas em placas de Petri de $15 \mathrm{~cm}$ de diâmetro, forradas com duas folhas de papel-filtro umedecido com $12 \mathrm{~mL}$ de solução-teste espe- 
cífica. As placas foram seladas com filme de PVC e incubadas a $27 \pm 0,5^{\circ} \mathrm{C}$ (Fanti, 2001).

$\mathrm{Na}$ avaliação da tolerância das sementes de paineira ao estresse hídrico, foram utilizadas soluções de manito $(\mathrm{PM}=182,17)$ preparadas de acordo com Parmar \& Moore (1968). Os potenciais osmóticos das soluções de manitol utilizados foram $0,0,-0,2,-0,4,-0,6,-0,8,-1,0$ $-1,2,-1,4$ e -1,6 MPa. Soluções de PEG 6000 foram preparadas de acordo com Villela et al. (1991) com os potenciais $0,0,-0,1,-0,2,-0,3,-0,4,-0,5,-0,6$ e $-0,7 \mathrm{MPa}$

As soluções salinas foram preparadas a partir da equação de Van't Hoff, citada por Salisbury \& Ross (1992). Os coeficientes isotônicos para os sais cloreto de sódio, cloreto de potássio e cloreto de cálcio são, respectivamente, $1,8,1,8$ e 2,4. Os potenciais osmóticos das soluções de $\mathrm{NaCl}, \mathrm{CaCl}_{2}$ e $\mathrm{KCl}$ utilizadas foram de $0,0,-0,2$, $-0,4,-0,6,-0,8,-1,0$ e $-1,2 \mathrm{MPa}$

Para que ficassem expostas a níveis constantes dos potenciais osmóticos das soluções, as sementes foram transferidas diariamente para outras placas de Petri forradas com papel-filtro recém-umedecido nas respectivas soluções.

A leitura do experimento foi realizada diariamente, sendo consideradas germinadas as sementes que apresentavam extensão radicular maior ou igual a $2 \mathrm{~mm}$ curvatura geotrópica positiva (Duran \& Tortosa, 1985) Os testes de germinação foram considerados finalizados quando todas as sementes já haviam germinado, ou quando as sementes remanescentes nas placas apresentavam-se deterioradas.

O cálculo de porcentagem de germinação foi realizado conforme Labouriau \& Agudo (1987) e a distribuição dos desvios foi analisada pelo teste de Bartlett. Os valores de porcentagem de germinação referentes aos experimentos de estresse hídrico e salino foram transformados em arco seno $(\% / 100)^{0,5}$ (Pimentel-Gomes, 2000).

Para cálculo de $\mathrm{P}_{50}$, que corresponde ao potencial osmótico em que ocorre redução da germinação para $50 \%$, foi utilizada a equação de ajuste $\mathrm{Y}=\mathrm{P}_{1} /[1+\exp$ $\left(\mathrm{P}_{2}-\left(\mathrm{P}_{3} . \mathrm{X}\right)\right]$, em que $\mathrm{Y}$ é a porcentagem de germinação e X é o potencial osmótico (MPa).

Foi utilizado o delineamento estatístico inteiramente casualizado, com quatro repetições. Para avaliação dos dados referentes ao estresse salino, utilizou-se o esquema fatorial de $3 \times 7$, três sais e sete potenciais osmóticos. $\mathrm{Na}$ ocorrência de interações significativas entre os fatores, foram feitos os desdobramentos. Quantos aos dados referentes ao estresse hídrico, utilizou-se um es- quema de 1x9, 1 agente e 9 potenciais osmóticos, para o manitol e 1x8, 1 agente e 8 potenciais, para o PEG 6000. A análise estatística foi realizada pelo teste $\mathrm{F}$ e, na comparação dos valores médios, utilizou-se o teste de Tukey a 5\% de probabilidade (Zar, 1996; Pimentel-Gomes, 2000).

\section{Resultados e Discussão}

Houve uma redução da viabilidade das sementes com a redução do potencial osmótico das soluções de PEG 6000 e manitol (Tabela 1). Um decréscimo significativo da porcentagem de germinação foi registrado a partir do potencial de $-0,5 \mathrm{MPa}$ para o PEG 6000 e -0,6 MPa para o manitol (Tabela 1). A menor tolerância ao estresse hídrico simulado foi apresentada pelos tratamentos com PEG 6000, em relação ao manitol. O limite máximo de tolerância ao PEG 6000 esteve entre -0,6 e -0,7 MPa e ao manitol, entre -1,4 e -1,6 MPa. A presença de diferentes valores de potencial osmótico quanto ao limite máximo de tolerância ao estresse hídrico simulado com os agentes PEG 6000 e manitol pode ser atribuída à permeabilidade diferencial do tegumento das

Tabela 1. Valores médios de germinação de sementes de paineira submetidas a soluções de diferentes potenciais osmóticos de PEG 6000 e manitol ${ }^{(1)}$

\begin{tabular}{cc}
$\begin{array}{c}\text { Potencial osmótico } \\
(\mathrm{MPa})\end{array}$ & $\begin{array}{c}\text { Germinação } \\
\left(\operatorname{arco~seno~}(\% / 100)^{0,5}\right)\end{array}$ \\
\hline PEG 6000 & $82,39 \mathrm{~A}$ \\
$-0,0$ & $80,36 \mathrm{~A}$ \\
$-0,1$ & $77,32 \mathrm{~A}$ \\
$-0,2$ & $71,40 \mathrm{~A}$ \\
$-0,3$ & $67,45 \mathrm{~A}$ \\
$-0,4$ & $43,53 \mathrm{~B}$ \\
$-0,5$ & $19,14 \mathrm{C}$ \\
$-0,6$ & - \\
$-0,7$ & \\
\hline Manitol & $82,39 \mathrm{~A}$ \\
$-0,0$ & $77,72 \mathrm{AB}$ \\
$-0,2$ & $75,51 \mathrm{AB}$ \\
$-0,4$ & $69,22 \mathrm{BC}$ \\
$-0,6$ & $63,67 \mathrm{CD}$ \\
$-0,8$ & $61,99 \mathrm{CD}$ \\
$-1,0$ & $54,42 \mathrm{D}$ \\
$-1,2$ & $26,33 \mathrm{E}$ \\
$-1,4$ & - \\
$-1,6$ & \\
\hline
\end{tabular}

${ }^{(1)}$ Médias seguidas por letras iguais, na mesma coluna, não diferem entre (1)Médias seguidas por letras iguais, na mesma coluna, não diferem entre
si pelo teste de Tukey a 5\% de probabilidade; os coeficientes de variasi pelo teste de Tukey a 5\% de probabilidade; os coeficientes de varia-
ção em relação à germinação para o agente PEG 6000 e manitol foram ção em relação à germinação para o age
de $10,58 \%$ e $7,78 \%$, respectivamente. 
sementes aos solutos de baixo peso molecular, o que, neste caso, teria favorecido a entrada de manitol nas sementes, reduzindo o verdadeiro efeito de seca. Além do alto peso molecular que impede a penetração através das membranas celulares, o PEG 6000 pode reduzir a disponibilidade de oxigênio em virtude de sua alta viscosidade, afetando o processo germinativo (Bradford, 1995).

Observou-se grande incidência de fungos quando as sementes de paineira foram expostas aos diferentes potenciais osmóticos das soluções de PEG 6000, reduzindo seu vigor e viabilidade. A hidratação da semente leva à liberação de solutos para o meio circundante, tais como açúcares, ácidos orgânicos, aminoácidos e vários íons, o que pode estimular o crescimento de patógenos, causando a deterioração das sementes (Bewley \& Black, 1994).

Entre as espécies que também não apresentaram limites elevados de tolerância ao estresse hídrico simulado com PEG 6000, pode-se citar Adenanthera pavonina L. (Fanti \& Perez, 1998) e Leucaena leucocephala (Lam.) de Wit. (Fonseca \& Perez, 1999). Quando utilizado o manitol, níveis de tolerância semelhantes aos verificados em paineira foram constatados em sementes de Prosopis juliflora (Sw.) D.C. (Perez, 1988), Bauhinia forficata L. (Fanti \& Perez, 1996) Leucaena leucocephala (Lam.) de Wit. (Cavalcante \& Perez, 1995).

A primeira e mais sensível resposta ao déficit hídrico é a redução da turgescência celular, que leva à diminuição do crescimento, uma vez que a divisão, alongamento e diferenciação celular são afetados por déficits

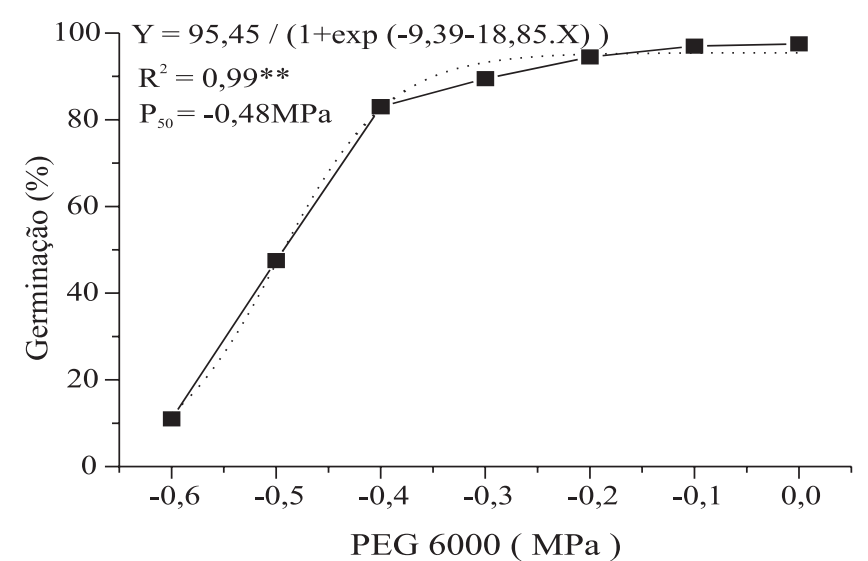

hídricos. Em condições não tão severas de déficit hídrico, o vegetal pode evitar a redução na turgescência celular iniciando medidas osmorregulatórias, como a síntese de compostos orgânicos e a conversão do amido em carboidratos solúveis, garantindo deste modo o influxo de água e a manutenção do volume da célula (Bradford, 1990; Larcher, 2000). O ajuste osmótico, ou seja, a diminuição do potencial osmótico pelo aumento real de solutos intracelulares, é um importante mecanismo para subsitir ao estresse hídrico (Bray, 1993).

Em sementes de paineira, a redução da germinação para $50 \%$ ocorreu em potencial osmótico de $-0,48 \mathrm{MPa}$ de PEG 6000, enquanto nas sementes mantidas em soluções de manitol esta redução foi constatada somente a -1,25 MPa, indicando, portanto, maior sensibilidade ao estresse hídrico induzido pelo PEG 6000 (Figura 1).

O estresse hídrico, em condições naturais, pode atuar de forma positiva no estabelecimento das espécies, pois provoca um atraso considerável no tempo de germinação das sementes. Dessa forma, a germinação é distribuída no tempo e no espaço, aumentando a probabilidade das plântulas encontrarem condições ambientais adequadas ao estabelecimento e desenvolvimento (Bewley \& Black, 1994).

A porcentagem de germinação não apresentou decréscimos significativos em presença dos sais $\mathrm{NaCl}, \mathrm{KCl}$ e $\mathrm{CaCl}_{2}$ até o potencial osmótico de $-0,4 \mathrm{MPa}$ mas, a partir de -0,6 MPa, reduções significativas na viabilidade foram registradas (Tabela 2). Nos potenciais osmóticos de - 0,8 e -1,0 MPa, observou-se decréscimos mais acentuados da porcentagem de germinação quando as sementes estavam embebidas nas soluções

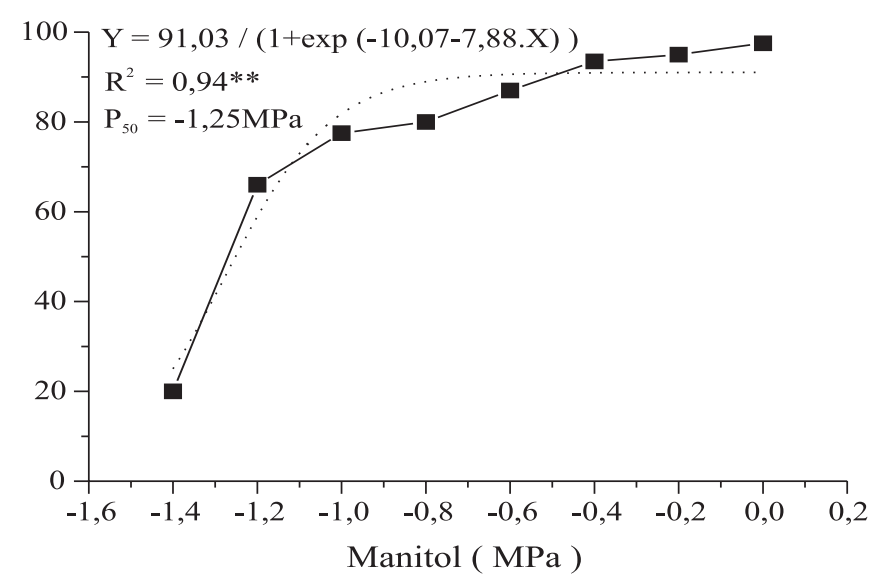

Figura 1. Valores experimentais ( - ) e esperados $(\cdots)$ de germinação de sementes de Chorisia speciosa submetidas a soluções de PEG 6000 e manitol com diferentes potenciais osmóticos. **Significativo a $1 \%$ de probabilidade. 
de $\mathrm{NaCl}$ e $\mathrm{CaCl}_{2}$, indicando maior sensibilidade a estes sais. O limite máximo de tolerância ao estresse salino apresentado pelas sementes de paineira foi o mesmo para os três sais avaliados e está situado entre -1,0 MPa e -1,2 MPa.

A redução na porcentagem de germinação e o atraso no início do processo germinativo com o aumento do estresse salino podem estar relacionados com a seca fisiológica produzida, pois quando existe aumento da concentração de sais no meio germinativo, há uma diminuição do potencial osmótico e, conseqüentemente, uma redução do potencial hídrico. Esta redução pode afetar a cinética de absorção de água pelas sementes (efeito osmótico), como também elevar a níveis tóxicos a concentração de íons no embrião (efeito tóxico) (Cramer et al., 1986; Tobe et al., 2000).

A inibição da mobilização das reservas, segundo Prisco et al. (1981), pode ser atribuída aos efeitos dos sais na síntese "de novo" e atividade das enzimas responsáveis pela hidrólise e translocação dos produtos hidrolizados dos tecidos de reserva para o eixo embrionário, afetando deste modo o processo germinativo.

Nos valores médios da porcentagem de germinação nos diferentes potenciais osmóticos das soluções de $\mathrm{NaCl}, \mathrm{KCl}$ e $\mathrm{CaCl}_{2}$, e a partir do ajuste destas curvas (linha pontilhada), pode-se observar o potencial osmótico em que há redução da germinação para 50\% (Figura 2). Nos sais $\mathrm{NaCl}, \mathrm{KCl}$ e $\mathrm{CaCl}_{2}$, verificou-se que a redução para $50 \%$ da germinação ocorre em potenciais osmóticos de aproximadamente -0,63 MPa, -0,87 MPa e - $0,65 \mathrm{MPa}$, respectivamente. A redução da germinação para $50 \%$, verificada em potencial osmótico mais negativo de KCL, pode indicar uma maior resistência aos efeitos desse sal.

Além do efeito osmótico e tóxico, a tolerância à salinidade envolve o grau de tolerância do protoplasma a um distúrbio no balanço iônico, associado ao estresse salino, o qual depende da espécie vegetal, do tecido e do vigor (Cramer et al., 1986; Larcher, 2000).

Em concetrações elevadas, os íons $\mathrm{Na}^{+} \mathrm{e} \mathrm{Cl}^{-}$causam intumescimento do protoplasma, afetando a atividade enzimática, causando alterações quantitativas e qualitativas no metabolismo, o que resulta em baixa produção de energia, distúrbios na assimilação do nitrogênio, alterações no padrão de aminoácidos e no metabolismo das proteínas (Freire, 2000). Além disto, excesso de $\mathrm{Na}^{+} \mathrm{e}$ de $\mathrm{Cl}^{-}$no protoplasma ocasiona distúrbios em relação ao balanço iônico de $\mathrm{K}^{+}$e $\mathrm{Ca}^{2+}$, bem como no efeito específico dos íons sobre as enzimas e membranas (Larcher, 2000). $\mathrm{O} \mathrm{Na}^{+}$pode competir com o $\mathrm{K}^{+}$no transporte de substâncias através da membrana plasmática, reduzindo sua integridade (Franco et al., 1999).

Os íons $\mathrm{Ca}^{2+}$ são essenciais na manutenção da integridade da membrana plasmática e contribuem para a diminuição do extravazamento de $\mathrm{K}^{+}$, o qual é responsável pela síntese de proteínas, amido e ativação de muitas enzimas no processo germinativo (Catalan et al., 1994; Franco et al., 1999).

A habilidade do protoplasma de tolerar altas concentrações de sal depende da compartimentalização seletiva dos íons que entram na célula. A maior parte dos íons provenientes dos sais acumulam-se nos vacúolos, processo que reduz a concentração de sais a que o citoplasma está submetido, com proteção do sistema de enzimas dos efeitos do estresse salino (Larcher, 2000). O equilíbrio osmótico entre o citoplasma e os diferentes compartimentos celulares, como o vacúolo, é mantido por meio da síntese de compostos orgânicos com atividade osmótica. Os carboidratos solúveis e os aminoácidos contribuem para a proteção das biomembranas e das proteínas em relação aos efeitos deletérios da alta concentração iônica (Franco et al., 1999; Larcher 2000).

Tabela 2. Valores médios de germinação $\left(\operatorname{arco}\right.$ seno $\left.(\% / 100)^{0,5}\right)$ de sementes de paineira submetidas ao estresse salino com soluções osmóticas de $\mathrm{NaCl}, \mathrm{KCl}$ e $\mathrm{CaCl}_{2}{ }^{(1)}$

\begin{tabular}{ccccc}
\hline Potencial osmótico $(\mathrm{MPa})$ & $\mathrm{NaCl}$ & $\mathrm{KCl}$ & $\mathrm{CaCl}_{2}$ & Médias \\
\hline 0,0 & $80,02 \mathrm{~A}$ & $80,02 \mathrm{~A}$ & $78,39 \mathrm{~A}$ & $79,48 \mathrm{~A}$ \\
$-0,2$ & $69,61 \mathrm{~A}$ & $76,57 \mathrm{~A}$ & $65,81 \mathrm{~A}$ & $70,67 \mathrm{AB}$ \\
$-0,4$ & $66,52 \mathrm{~A}$ & $74,51 \mathrm{~A}$ & $69,71 \mathrm{~A}$ & $70,25 \mathrm{~B}$ \\
$-0,6$ & $47,14 \mathrm{~B}$ & $63,98 \mathrm{AB}$ & $48,79 \mathrm{~B}$ & $53,31 \mathrm{C}$ \\
$-0,8$ & $29,15 \mathrm{C}$ & $55,14 \mathrm{~B}$ & $31,99 \mathrm{C}$ & $38,77 \mathrm{D}$ \\
$-1,0$ & $18,65 \mathrm{C}$ & $29,30 \mathrm{C}$ & $10,49 \mathrm{D}$ & $19,49 \mathrm{E}$ \\
$-1,2$ & - & - & - & - \\
\hline Média & $51,85 \mathrm{~b}$ & $63,26 \mathrm{a}$ & $50,87 \mathrm{~b}$ & \\
\hline${ }^{(1)}$ Médias seguidas pelas mesmas letras, maiúsculas nas colunas e minúsculas nas linhas, não diferem entre si pelo teste de Tukey a $5 \%$ de
\end{tabular}

probabilidade; os coeficientes de variação em relação à germinação para o $\mathrm{NaCl}, \mathrm{KCl}$ e $\mathrm{CaCl}_{2}$ foram de $14,86 \%, 12,49 \%$ e $13,48 \%$, respectivamente. 
Segundo Larcher (2000), um grau moderado de resistência ao sal em plantas é útil na tentativa de utilização de solos afetados por sais em regiões secas. Considerando que aproximadamente um terço da superfície
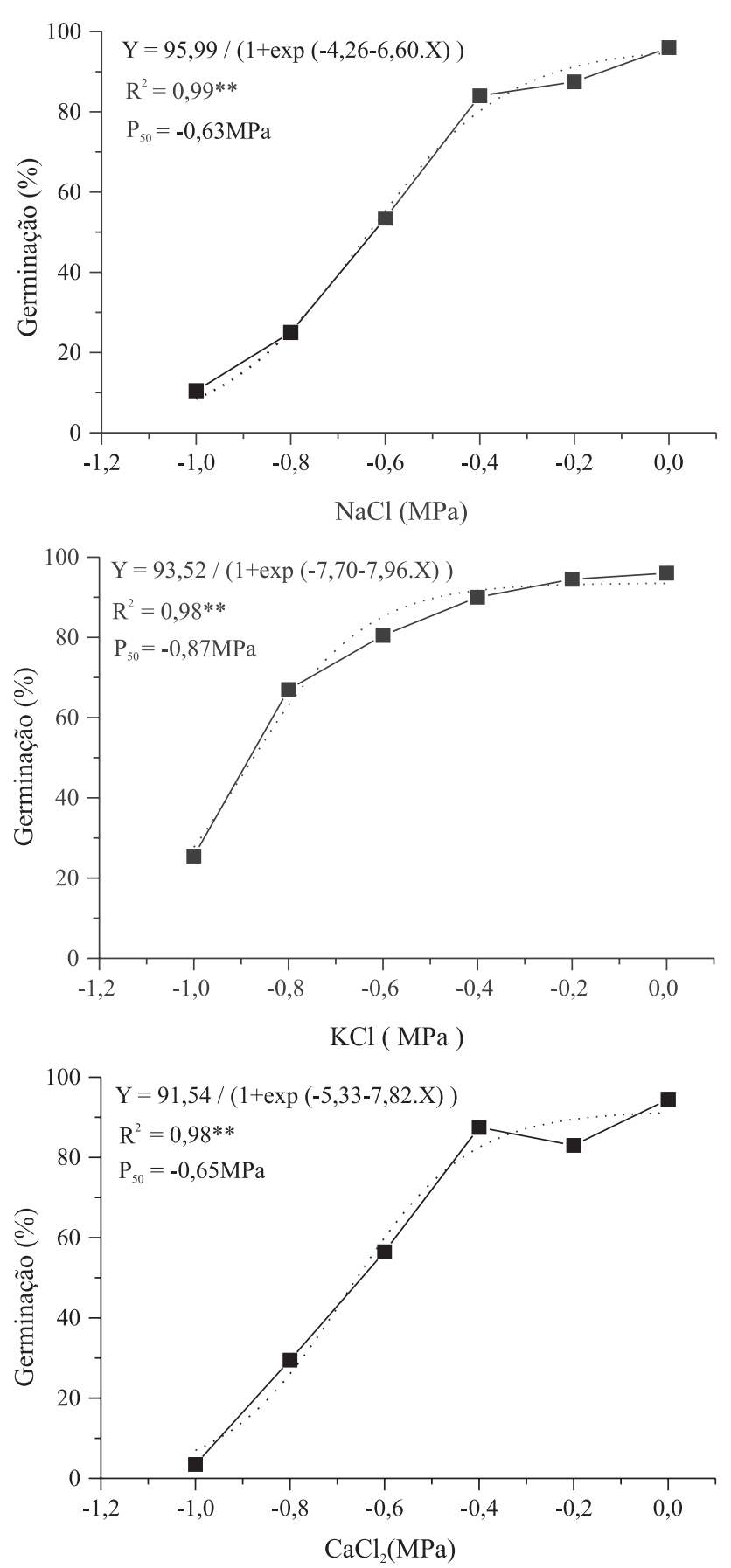

Figura 2. Valores experimentais (一) e esperados (....) de germinação de sementes de Chorisia speciosa submetidas soluções de $\mathrm{NaCl}, \mathrm{KCl}$ e $\mathrm{CaCl}_{2}$ com diferentes potenciais osmóticos. **Significativo a $1 \%$ de probabilidade. terrestre do planeta é árida ou semi-árida, e que dessa área estima-se que metade seja afetada por sais, é importante o investimento em pesquisas que visem à identificação espécies capazes de germinar e sobreviver satisfatoriamente nessas condições.

Sementes de paineira não apresentaram um limite elevado de tolerância ao estresse salino, podendo esta espécie ser classificada como glicófita, com moderada tolerância aos sais $\mathrm{NaCl}, \mathrm{KCl}$ e $\mathrm{CaCl}_{2}$.

Entre as espécies que apresentaram limite de tolerância ao estresse salino semelhante ao verificado em sementes de paineira pode-se citar Leucaena leucocephala (Lam.) de Wit. (Cavalcante \& Perez, 1995), Bauhinia forficata L. (Fanti \& Perez, 1996), Copaifera langsdorffii Desf. (Jeller \& Perez ,1997), Adenanthera pavonina L. (Fanti \& Perez, 1998) e Plathymenia reticulata Benth. (Miranda, 1999).

\section{Conclusões}

1. O decréscimo dos níveis de potencial osmótico das soluções de PEG 6000, manitol e dos sais no meio germinativo provoca redução da viabilidade e vigor.

2. O uso de PEG 6000 reduz o limite máximo de tolerância ao estresse hídrico.

3. As sementes de paineira são mais sensíveis ao $\mathrm{NaCl}$ e $\mathrm{CaCl}_{2}$ em relação ao $\mathrm{KCl}$.

4. A espécie Chorisia speciosa St. Hil. pode ser classificada como glicófita com moderada tolerância aos sais $\mathrm{NaCl}, \mathrm{KCl}$ e $\mathrm{CaCl}_{2}$.

\section{Referências}

AGBOOLA, D.A. Effect of saline solutions and salt stress on seed germination of some tropical forest tree species. Revista de Biología Tropical, v.46, p.1109-1115, 1998.

BEWLEY, J.D.; BLACK, M. Seeds: physiology of development and germination. New York: Plenum Press, 1994. 445p.

BRADFORD, K.J. A water relation analysis of seed germination rates. Plant Physiology, v.94, p.840-849, 1990.

BRADFORD, K.J. Water relations in seed germination. In: KIGEL, J.; GALILI, G. (Ed.). Seed development and germination. New York: Marcel Dekker Inc., 1995. p.351-396.

BRAY, E.A. Molecular responses to water deficit. Plant Physiology, v.103, p.1035-1040, 1993

CARVALHO, N.M.; NAKAGAWA, J. Sementes: ciência, tecnologia e produção. 4.ed. Jaboticabal: Funep, 2000. 588p.

CARVALHO, P.E.R. Espécies florestais brasileiras: recomendações silviculturais, potencialidades e uso da madeira. Colombo: Embrapa-CNPF, 1994. 640p. 
CATALAN, L.; BALZARINI, Z.; ALESNIK, E.; SERENO, R.; KARLIN, U. Effects of salinity on germination and seedling growth of Prosopis flexuosa (D.C.). Forest Ecology and Management, v.63, p.347-357, 1994.

CAVALCANTE, A.M.B.; PEREZ, S.C.J.G.A. Efeitos dos estresses hídrico e salino sobre a germinação de sementes de Leucaen leucocephala (Lam.) de Wit. Pesquisa Agropecuária Brasileira v.30, p.281-289, 1995.

CLARK, S.M.; SCOTT, D.J. Effects of carboxin, benomyl an captan on the germination of wheat during the postharvest dormancy period. Seed Science and Technology, v.10, p.87-94, 1982.

CRAMER, G.R.; LÄUCHLI, A.; EPSTEIN, E. Effects of $\mathrm{NaCl}$ and $\mathrm{CaCl}_{2}$ on ion activities in complex nutrient solutions and root growth of cotton. Plant Physiology, v.81, p.792-797, 1986.

DURAN, J.M.; TORTOSA, M.E. The effect of mechanical and chemical scarification on germination of charlock Sinapsis arvensis L. seed. Seed Science and Technology, v.13, p.155-163, 1985.

FANTI, S.C. Aspectos da germinação e efeitos do condicionamento osmótico em sementes de paineira (Chorisia speciosa St. Hil. Bombacaceae). 2001. 145p. Tese (Doutorado) - Universidade Federal de São Carlos, São Carlos.

FANTI, S.C.; PEREZ, S.C.J.G.A. Efeitos do estresse hídrico salino na germinação de Bauhinia forficata Link. Revista Ceres, v.43, p.654-662, 1996.

FANTI, S.C.; PEREZ, S.C.J.G.A. Efeitos do estresse hídrico, salino e térmico no processo germinativo de sementes de Adenanthera pavonina L. Revista Brasileira de Sementes, v.20, p.167-177, 1998.

FERREIRA, L.G.R.; REBOUÇAS, M.A.A. Influência da hidratação /desidratação de sementes de algodão na superação dos efeitos d salinidade na germinação. Pesquisa Agropecuária Brasileira, v.27, p.609-615, 1992.

FONSECA, S.C.L.; PEREZ, S.C.J.G.A. Efeito do estresse hídrico e interferência de diferentes profundidades de plantio na germinação de sementes de Leucaena leucocephala (Lam) de wit. Revista Ceres, v.46, p.371-381, 1999.

FRANCO, O.L.; ENÉAS-FILHO, J.; PRISCO, J.T.; GOMES FILHO, E Effects of $\mathrm{CaCl}_{2}$ on the growth and osmoregulato accumulation in $\mathrm{NaCl}$ stressed cowpea seedlings. Revista Brasileir de Fisiologia Vegetal, v.11, p.145-151, 1999.

FREIRE, A.L. de O. Fixação do nitrogênio, crescimento e nutrição mineral de leucena sob condições de salinidade. 2000. 92p Tese (Doutorado) - Universidade Estadual Paulista, Jaboticabal. HARDEGREE, S.P.; EMMERICH, W.E. Seed germination response to polyethylene glycol solution depth. Seed Science and Technology, v.22, p.1-7, 1994.

JELLER, H.; PEREZ, S.C.J.G.A. Efeito da salinidade e semeadura en diferentes profunfidades na viabilidade e no vigor de Copaifer langsdorffii. Revista Brasileira de Sementes, v.19, p.219-225, 1997.
LABOURIAU, L.G.; AGUDO, M. On the physiology of germination in Salvia hispanica L. temperature effects. Anais da Academia Brasileira de Ciências, v.59, p.37-56, 1987.

LARCHER, W. Ecofisiologia vegetal. Trad. de C.H.B.A. Prado. São Carlos: Rima, 2000. 531p.

LUCCA, A.; REIS, M.S. Considerações sobre a influência do potencial hídrico e do condicionamento osmótico na qualidade fisiológica de sementes de soja. Informativo Abrates, v.5, p.42-50, 1995

LORENZI, H. Árvores brasileiras: manual de identificação e cultivo de plantas arbóreas nativas do Brasil. Nova Odessa: Plantarum, 1992. de plan

MAYER, A.M.; POLJAKOFF-MAYBER, A. The germination of seeds. 4.ed. Great Britain: Pergamon Press, 1989. 270p.

MIRANDA, A.R. de Fenologia, aspectos ecofisiológicos da germinação de sementes de Plathymenia reticulata Benth. Fabaceae-Mimosoideae. 1999. 184p. Tese (Doutorado) Universidade Federal de São Carlos, São Carlos.

PARMAR, M.T.; MOORE, R.P. Carbowax 6000, mannitol and sodium choride for simulating drought conditions in germination of corn (Zea mays L.) of strong and weak vigor. Agronomy Journal, v.30, p.192-195, 1968

PEREZ, S.C.J.G.A. Aspectos ecofisiológicos da germinação de sementes de Prosopis juliflora (Sw.) DC. 1988. 214p. Tese (Doutorado) - Universidade Federal de São Carlos, São Carlos.

PIMENTEL-GOMES, F. Curso de Estatística Experimental. 14.ed. Piracicaba: Escola Superior de Agricultura Luiz de Queiroz, 2000. 477p.

PRISCO, J.T.; ENEAS FILHO, J.; GOMES FILHO, E. Effect of $\mathrm{NaCl}$ salinity on cotyledon starch mobilization during germination of Vigna unguiculata (L.) Walp seeds. Revista Brasileira de Botânica, v.4, p.63-71, 1981.

SALISBURY, F.B.; ROSS, C.W. Plant physiology. 4th ed. Belmont: Wadsworth Publishing Company, 1992. 682p.

SERRATO VALENTI, G.; FERRO, M.; FERRARO, D.; RIVEROS, F. Anatomical changes in Prosopis tamarugo Phil. seedlings growing at different levels of $\mathrm{NaCl}$ salinity. Annals of Botany, v.68, p.47-53, 1991.

TOBE, K.; LI, X.; OMASA, K. Seed germination and radicle growth of a halophyte, Kalidium caspicum (Chenopodiaceae). Annals of Botany, v.85, p.391-396, 2000.

TORRES, S.B.; VIERA, E.L.; MARCOS-FILHO, J. Efeitos do estresse hídrico na germinação e no desenvolvimento de plântulas de pepino. Revista Brasileira de Sementes, v.21, p.59-63, 1999.

VILLELA, F.A.; DONI-FILHO, L.; SEQUEIRA, E.L. Tabela de potencial osmótico em função da concentração de polietileno glicol 6000 e da temperatura. Pesquisa Agropecuária Brasileira, v.26, p.1957-1968, 1991.

ZAR, J.H. Bioestatistical analysis. Englewood Cliffs: Prentice Hall Inc., 1996. 620p.

Recebido em 10 de março de 2004 e aprovado em 20 de maio de 2004 